\title{
Testing the Kerr metric using X-ray reflection spectroscopy: spectral analysis of GX 339-4
}

\author{
Jingyi Wang-Ji, ${ }^{1,2}$ Askar B. Abdikamalov, ${ }^{1}$ Dimitry Ayzenberg, ${ }^{1}$ Cosimo Bambi, ${ }^{1,3}, *$ \\ Thomas Dauser, ${ }^{4}$ Javier A. García, ${ }^{5,4}$ Sourabh Nampalliwar, ${ }^{3}$ and James F. Steiner ${ }^{2}$ \\ ${ }^{1}$ Center for Field Theory and Particle Physics, Department of Physics, Fudan University, 200438 Shanghai, China \\ ${ }^{2}$ MIT Kavli Institute for Astrophysics and Space Research, MIT, Cambridge, MA 02139, USA \\ ${ }^{3}$ Theoretical Astrophysics, Eberhard-Karls Universität Tübingen, 72076 Tübingen, Germany \\ ${ }^{4}$ Remeis Observatory \& ECAP, Universität Erlangen-Nürnberg, 96049 Bamberg, Germany \\ ${ }^{5}$ Cahill Center for Astronomy and Astrophysics, California Institute of Technology, Pasadena, CA 91125, USA
}

\begin{abstract}
Reflection features commonly observed in the X-ray spectrum of black holes can be used to probe the strong gravity region around these objects. In this paper, we extend previous work in the literature and we employ the first full emission model for relativistic reflection in non-Kerr spacetime to test the Kerr black hole hypothesis. We analyze a composite spectrum from the detector RXTE PCU-2 of the stellar-mass black hole GX 339-4 in its brightest hard state. With an unprecedented sensitivity of $\sim 0.1 \%$ and 40 million counts to capture the faint features in the reflection spectrum, we demonstrate that it is possible with existing data and an adequate model to place constraints on the black hole spin $a_{*}$ and the Johannsen deformation parameter $\alpha_{13}$. For a Kerr black hole of general relativity, $\alpha_{13}=0$. Our measurement, which should be regarded as principally a proof of concept, is $a_{*}=0.92_{-0.12}^{+0.07}$ and $\alpha_{13}=-0.76_{-0.60}^{+0.78}$ with a $90 \%$ confidence level and is consistent with the hypothesis that the compact object in GX 339-4 is a Kerr black hole.
\end{abstract}

\section{INTRODUCTION}

Einstein's theory of general relativity (GR hereafter) was proposed at the end of 1915 [1] and is currently the standard framework for the description of gravitational fields and the chrono-geometrical structure of spacetime. While largely successful, application of Einstein's theory to our Universe has led to some unexplained phenomena, e.g., dark energy. Even on purely theoretical grounds, there are issues like the presence of singularities and the difficulty in finding a good theory of quantum gravity. A number of extensions and modifications of GR have been proposed to deal with these issues. Most of these alternative theories have the same predictions as Einstein's gravity for weak fields and present deviations only when gravity becomes strong ${ }^{1}$. Thanks to the advent of new observational facilities, there is today an increasing interest in the possibility of testing GR in the strong gravity regime [3-8]. Black holes are the most interesting candidates for such strong gravity tests.

Black holes are extremely compact objects in which matter has collapsed to a singularity. Today we have a body of observational evidence strongly supporting the existence of dark and compact objects within our Galaxy and across the Universe which can be interpreted as the black holes of Einstein's theory. They are found in various scenarios, like stellar collapse [9], within galactic centers [10], and mergers $[11,12]$. In 4-dimensional GR, the

\footnotetext{
* Corresponding author: bambi@fudan.edu.cn

${ }^{1}$ Over the past century, there have been significant efforts to test the theory in weak gravitational fields, mainly with experiments in the Solar System and radio observations of binary pulsars [2]. GR has been highly successful in this regime and it is desirable that alternative theories match with GR in this regime.
}

only stationary and asymptotically flat vacuum (electrovacuum) black hole spacetime with a regular exterior region is described by the Kerr (Kerr-Newman) solution $[13,14]$. It is also intriguing that these black holes are extremely simple objects, being defined by not more than three parameters: mass $M$, spin angular momentum $J$, and electric charge $Q$ [15-18].

It is a remarkable fact that the spacetime metric around astrophysical black holes is well described by the Kerr solution. As soon as a black hole is formed, initial deviations from the Kerr metric are quickly radiated away with the emission of gravitational waves [19, 20]. The equilibrium electric charge is extremely small for macroscopic objects and completely negligible for the metric of the spacetime [21]. Accretion disks typically have a mass of many orders of magnitude smaller than the central object and their impact on the background metric can be safely ignored $[22,23]$. Eventually, macroscopic deviations from the Kerr spacetime would only be possible in the presence of new physics, such as classical extensions of GR [24], macroscopic quantum gravity effects [25-27], or in the presence of exotic matter [28, 29]. These deviations would affect the spacetime in a variety of ways and by probing the spacetime geometry, one can identify the deviations and thus test the extensions and modifications of GR.

Currently, there are two approaches for probing the spacetime geometry: electromagnetic radiation [30-32] and gravitational waves $[33,34]$. With the electromagnetic approach, currently there are two leading techniques to probe the spacetime geometry around a black hole: the study of the thermal spectrum of thin disks (continuum-fitting method) [35-37] and the analysis of the relativistically smeared reflection spectrum of thin disks (reflection method, often called the iron line method) [38-40]. Both techniques have been developed 
for measuring black hole spins under the assumption of Kerr background and can be naturally extended for testing the Kerr metric [41-48]. The reflection method has some advantages over the study of the thermal spectrum. The former can be easily applied to both stellar-mass and supermassive black holes, while the latter is only observable in stellar-mass black hole systems. The simple shape of the thermal continuum is advantageous for measuring spin (when assuming a Kerr geometry and fitting two parameters), but not suitable for probing higher order structures including searches for deviations from Kerr. In contrast the reflection spectrum has a distinct structure with a number of prominent features, e.g. fluorescent lines and absorption edges. Thus, in the presence of high quality data and the correct astrophysical model, the reflection method is potentially quite a powerful tool to constrain the metric around black holes (see, for instance, Refs. $[49,50])$. The current limitation of the technique is mainly the uncertainty in the astrophysical model (e.g., the coronal/disk geometry and dynamics of the corona).

The content of the paper is as follows. In Section II, we review parametrically deformed Kerr metrics and describe a non-Kerr metric that we implemented in our reflection model. We review the technique of X-ray reflection spectroscopy and our reflection model in Section III. Section IV describes the observations, data reduction, and outlines our procedure for combining the individual spectra into the composite spectrum. Our fitting procedure and results of the constraint of the deformation parameter and the spin parameter appear in Section V. We draw our conclusions in Section VI.

Throughout the paper, we employ units in which $G_{\mathrm{N}}=$ $c=1$ and the convention of a metric with signature $(-+$ $++)$.

\section{TESTING THE KERR PARADIGM}

There are two natural strategies to test the Kerr black hole hypothesis. In the top-down approach, we begin with a specific modified theory of gravity which has solutions mimicking black holes. These solutions' differences from the Kerr solution are parametrized by one or more deformation parameters. In this approach, testing astrophysical data for the presence of deformation parameters amounts to testing the specific modified theory. This approach is the most logical, but it typically presents two problems. Firstly, there are a large number of modified theories of gravity and there is no clear preference for any one of them, so the search for deformation parameters has to be repeated for every theory. Secondly, black hole solutions are known in very few modified theories of gravity, and in many of those only the non-rotating and the slow-rotating solutions, while fast rotating black hole solutions, preferred observationally, are known only in exceptional cases.

In the bottom-up approach, we consider a phenomenological parameterization of the Kerr metric, which in principle should be able to describe the spacetime of any possible black hole in any possible gravity theory (in practice, bottom-up metrics describe black hole metrics with a range that encompasses a variety of, but not all, alternative theories). A number of deformation parameters are used to quantify possible deviations from the Kerr metric, and analysis of astrophysical data constrains potential deviations from the Kerr solution in a model-independent way.

The bottom-up approach is analogous to the Parametrized Post-Newtonian (PPN) formalism [2] that has been successfully employed to test the Schwarzschild solution in the weak field limit with Solar System experiments over the past several decades. Within the PPN framework, we write the most general static, spherically symmetric, and asymptotically flat metric in terms of the expansion parameter $M / r$, where $M$ is the mass of the central object and $r$ is some radial coordinate. When arranged in the Schwarzschild coordinates, the line element reads

$$
\begin{aligned}
d s^{2}= & -\left[1-\frac{2 M}{r}+(\beta-\gamma) \frac{2 M^{2}}{r^{2}}+\ldots\right] d t^{2} \\
& +\left(1+\gamma \frac{2 M}{r}+\ldots\right) d r^{2}+r^{2} d \theta^{2} \\
& +r^{2} \sin ^{2} \theta d \phi^{2}
\end{aligned}
$$

The second term in $g_{t t}$, i.e. $2 M / r$, is required to recover the Newtonian limit. $\beta$ and $\gamma$ are free parameters to be measured by experiments. The only spherically symmetric vacuum solution of Einstein's equations is the Schwarzschild metric and it requires $\beta=\gamma=1$. Other theories of gravity may have a different spherically symmetric vacuum solution, and in this case $\beta$ and $\gamma$ may not be exactly 1 . Current observational data in the Solar System provide the following constraints on $\beta$ and $\gamma[51,52]$

$$
|\beta-1|<2.3 \cdot 10^{-4}, \quad|\gamma-1|<2.3 \cdot 10^{-5},
$$

confirming the validity of the Schwarzschild solution in the weak field limit within the precision of current observations.

There are several proposals for bottom-up metrics, the most extensively employed ones are the JohannsenPsaltis metric [53], the Johannsen metric [54], and the Konoplya-Rezzolla-Zhidenko metric [55]. Here we adopt the Johannsen metric to describe the spacetime geometry. Its most important properties are that: $i$ ) the metric is regular (no naked singularities or closed time-like curves) everywhere on and outside of the event horizon, just like the Kerr metric, and ii) it was explicitly shown that it is able to recover some black hole solutions in alternative theories of gravity for suitable choices of the free parameters. In Boyer-Lindquist coordinates, the line 
element of the Johannsen metric reads [54]

$$
\begin{aligned}
d s^{2}= & -\frac{\tilde{\Sigma}\left(\Delta-a^{2} A_{2}^{2} \sin ^{2} \theta\right)}{B^{2}} d t^{2} \\
& -\frac{2 a\left[\left(r^{2}+a^{2}\right) A_{1} A_{2}-\Delta\right] \tilde{\Sigma} \sin ^{2} \theta}{B^{2}} d t d \phi \\
& +\frac{\tilde{\Sigma}}{\Delta A_{5}} d r^{2}+\tilde{\Sigma} d \theta^{2} \\
& +\frac{\left[\left(r^{2}+a^{2}\right)^{2} A_{1}^{2}-a^{2} \Delta \sin ^{2} \theta\right] \tilde{\Sigma} \sin ^{2} \theta}{B^{2}} d \phi^{2}
\end{aligned}
$$

where

$$
\begin{aligned}
& a=J / M, \quad B=\left(r^{2}+a^{2}\right) A_{1}-a^{2} A_{2} \sin ^{2} \theta, \\
& \tilde{\Sigma}=\Sigma+f, \Sigma=r^{2}+a^{2} \cos ^{2} \theta \\
& \Delta=r^{2}-2 M r+a^{2} .
\end{aligned}
$$

and the four free functions $f, A_{1}, A_{2}$, and $A_{5}$ are

$$
\begin{aligned}
& f=\sum_{n=3}^{\infty} \epsilon_{n} \frac{M^{n}}{r^{n-2}}, \\
& A_{1}=1+\sum_{n=3}^{\infty} \alpha_{1 n}\left(\frac{M}{r}\right)^{n}, \\
& A_{2}=1+\sum_{n=2}^{\infty} \alpha_{2 n}\left(\frac{M}{r}\right)^{n}, \\
& A_{5}=1+\sum_{n=2}^{\infty} \alpha_{5 n}\left(\frac{M}{r}\right)^{n} .
\end{aligned}
$$

The metric elements depend on the mass and spin of the black hole as well as on four free functions that measure potential deviations from the Kerr solution. The first order deformation parameters in these free functions are $\epsilon_{3}, \alpha_{13}, \alpha_{22}$, and $\alpha_{52}$. This metric exactly reduces to the Kerr solution for $\epsilon_{3}=\alpha_{13}=\alpha_{22}=\alpha_{52}=0$.

In the Kerr metric, the condition for the existence of an event horizon is $\left|a_{*}\right| \leq 1$, where $a_{*}=a / M=J / M^{2}$ is the dimensionless spin parameter. If $\left|a_{*}\right|>1$, there is no horizon and the singularity at the origin $r=0$ is naked. In the Johannsen spacetime, we still have the condition $\left|a_{*}\right| \leq 1$. Moreover, in order to exclude a violation of Lorentzian signature or the existence of closed time-like curves in the exterior region, we have to impose that the metric determinant is always negative and that $g_{\phi \phi}$ is never negative for radii larger than the radius of the event horizon. These conditions lead to the following constraints on the first-order deformation parameters [54]

$$
\begin{gathered}
\alpha_{13}, \epsilon_{3} \geq-\left(1+\sqrt{1-a_{*}^{2}}\right)^{3}, \\
\alpha_{22}, \alpha_{52} \geq-\left(1+\sqrt{1-a_{*}^{2}}\right)^{2} .
\end{gathered}
$$

In the present work, as a first step we only explore a nonvanishing $\alpha_{13}$, and we set to zero all other deformation

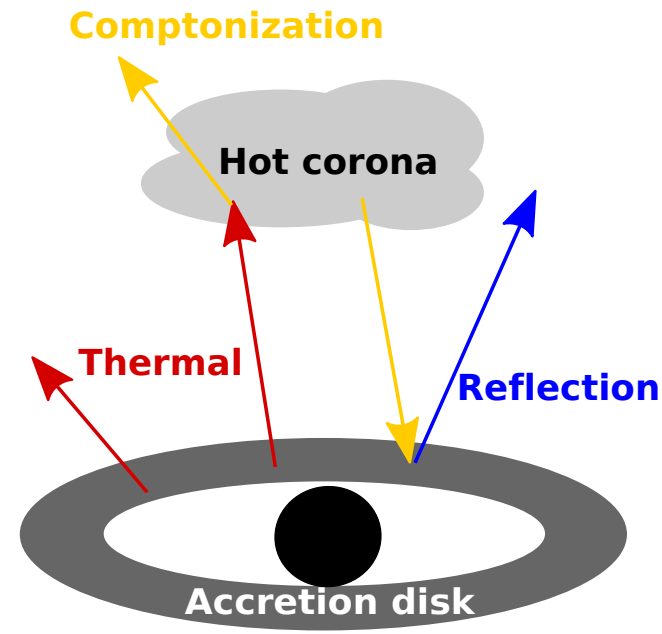

FIG. 1. A cartoon illustrating the process which gives rise to reflection emission from a disk-coronal geometry. The three main spectral components are indicated by different colored arrows. Thermal component: multi-temperature black body radiation from the disk. Comptonization component: inverse Compton scattering of the thermal photons off free electrons in a hot plasma cloud called the "corona". Reflection component: the Comptonized photons illuminate the disk, and interactions including Compton scattering and photoelectric absorption generate the reflection emission. See the text for more details.

parameters. Since the metric is singular for $B=0$, we impose that $B$ never vanishes for radii larger than the event horizon, and we obtain the following constraint

$$
\alpha_{13} \geq-\frac{1}{2}\left(1+\sqrt{1-a_{*}^{2}}\right)^{4}
$$

which is stronger than the requirement in Eq. (6).

\section{X-RAY REFLECTION SPECTROSCOPY}

The standard picture for an accreting black hole system involves an accretion disk and a compact corona of hot electrons (see Fig. 1). The accretion disk emits as a blackbody locally, and as a multi-temperature blackbody when integrated radially (see, e.g., Ref. [56]). The temperature of the disk depends on the black hole mass, the mass accretion rate, the position in the disk, and at some level on the spin and possible deformation parameters. For a black hole accreting at $\sim 10 \%$ of its Eddington limit, the thermal emission of the inner part of the accretion disk is in the soft X-ray band $(\sim 1 \mathrm{keV})$ for stellarmass black holes and in the optical/UV band $(1 \sim 10 \mathrm{eV})$ for the supermassive ones. Inverse Compton scattering of the thermal photons from the accretion disk off free electrons in a hotter $(\sim 100 \mathrm{keV})$ plasma cloud, called the "corona", generates a Comptonization component, which is often modeled with a cut-off power-law spectrum. A 
fraction of the Comptonized photons illuminate the disk. The integration of these photons with the material of the accretion disk, which mainly includes Compton scattering and photoelectric absorption followed by fluorescent line emission or Auger de-excitation, produces the reflection spectrum.

The reflection spectrum is a rich combination of radiative recombination continua, fluorescent lines (most notably the iron $\mathrm{K}$ complex at $6 \sim 8 \mathrm{keV}$ ), absorption edges, and a Compton hump at $>10 \mathrm{keV}$. This radiation carries information on the physical composition of the accretion disk and on the strong gravitational field around the black hole. The Fe K emission line (and other fluorescent lines) are broadened and skewed by relativistic effects (Doppler effects, gravitational redshift, and light bending). To illustrate the relativistic effect of the black hole, we show in Fig. 2 left panel the broadened iron lines for three representative values of the black hole spin, viz., $-0.99,0$ and 0.99, while keeping all deformations zero. The clear change in the shape of the line for different spin shows that by fitting for the line, one can recover the spin of the black hole. This has been a standard technique for measuring black hole spin for a decade. In the right panel of the same figure, we show the lines, keeping spin constant, for three representative values of the Johannsen deformation parameter $\alpha_{13}$. $\alpha_{13}$ too changes the shape of the line and therefore, reflection spectroscopy can also be used to estimate the deviation from Kerr (see Ref. [57] for the effect of other Johannsen deformation parameters on the iron line shape).

We employ one of the standard software systems for analyzing X-ray spectral data, XsPEC [58]. The reflection model most widely used in the past for both general application and measuring black hole spins is REFLIONX [59]. Recently, an improved reflection model, RELXILL, has been developed. It is based on the reflection code XILLVER [60-62], and the relativistic lineemission code RELLINE [63-65]. Compared to REFLIONX, RELXILL incorporates a superior treatment of radiative transfer and Compton redistribution, and it allows for the angular dependence of the reflected spectrum. Furthermore, by implementing the routines of the photoionization code XSTAR [66], RELXILL provides an improved calculation of the ionization balance. At the same time, limitations of the model include assuming that the density of the disk is independent of vertical height and radius, that ionization is constant over radius, that the illuminating radiation strikes the disk at a fixed angle of $45^{\circ}$, and that, apart from Fe, all the elemental abundances are assumed to be Solar. The results presented in this paper are derived using an extension of RELXILL to model the relativistically-blurred reflection component from the inner disk and XILLVER to model a distant reflector. The disk-coronal geometry is largely unknown and unconstrained, so we follow the usual assumption of adopting a (broken) power-law emissivity profile for the reflection emission as a pragmatic option.

By calculating the transfer function for a generic stationary, axisymmetric, and asymptotically flat black hole spacetime, an extended relativistic reflection model RELXILL_NK has been constructed (see Ref. [57] for details on the model and Refs. [8, 50] for some results). Presently, RELXILL_NK includes $\alpha_{13}, \alpha_{22}, \epsilon_{3}$ from the Johannsen metric and the conformal gravity parameter $L$ from the black hole solutions of $[67,68]$ as the deformation parameters. The analysis in this paper is limited to $\alpha_{13}$, which has the largest impact on the shape of reflection spectrum among the deformation parameters of the Johannsen metric; this means that the other deformation parameters are set to be zero.

\section{OBSERVATIONS, DATA REDUCTION, AND COMBINING SPECTRA}

We use observations from the X-ray mission Rossi $X$ ray Timing Explorer ( $R X T E$ ) which has the principal detector the Proportional Counter Array (PCA) on board containing five Proportional Counter Units (PCUs), with sensitivity from $2 \sim 60 \mathrm{keV}$. Despite the limited spectral resolution of the instrument $(17 \%$ at $6 \mathrm{keV})$ the archive of PCA data amassed during the RXTE mission (1995 to $\sim 2012$ ) continues to be excellent for the synoptic study of stellar-mass black holes. A few-dozen bright black holes were observed daily during their outburst cycles with typical exposure times of a few ks.

RXTE/PCA has several advantages. Prominently, it is free from the problematic effects of pileup, which is commonly a serious problem in analyzing and interpreting data for bright sources obtained using CCD detectors. Another attribute of the PCA, which has only recently been suprassed by NuSTAR, is its high-energy sensitivity, which allows observations of both the Fe $\mathrm{K}$ region and the Compton hump using a single detector. By implementing a calibration tool called PCACORR, the sensitivity of the RXTE PCA detector to faint spectral features (such as the $\mathrm{Fe} \mathrm{K}$ line/edge) is increased by up to an order of magnitude [69].

We test our non-Kerr model on GX 339-4 using the observations previously analyzed in Ref. [70], because of the high signal-to-noise ratio of the data and because we can then compare the results here with those in [70]. For simplicity, here we only consider the brightest data from GX 339-4's hard state. We analyze just the data collected using the best-calibrated detector, PCU-2, which also provides the richest data set. In order to boost signalto-noise ratio, we combine a composite PCU-2 hard-state spectrum of GX 339-4 with a total exposure time of $46 \mathrm{ks}$, which is a summation of 23 individual exposures taken during the 2002 outburst, all of them corresponding to roughly the same source intensity $\left(L / L_{\mathrm{Edd}}=0.17\right.$ assuming a distance of $8 \mathrm{kpc}$ and a black hole mass of $10 M_{\odot}$ ). The total number of source counts is 40 million and the counts-per-keV in the continuum at $6.4 \mathrm{keV}$ is $4.4 \mathrm{mil}-$ lion, while the total number of counts in the Fe $\mathrm{K}$ line region $(3 \sim 10 \mathrm{keV})$ is 28 million. 

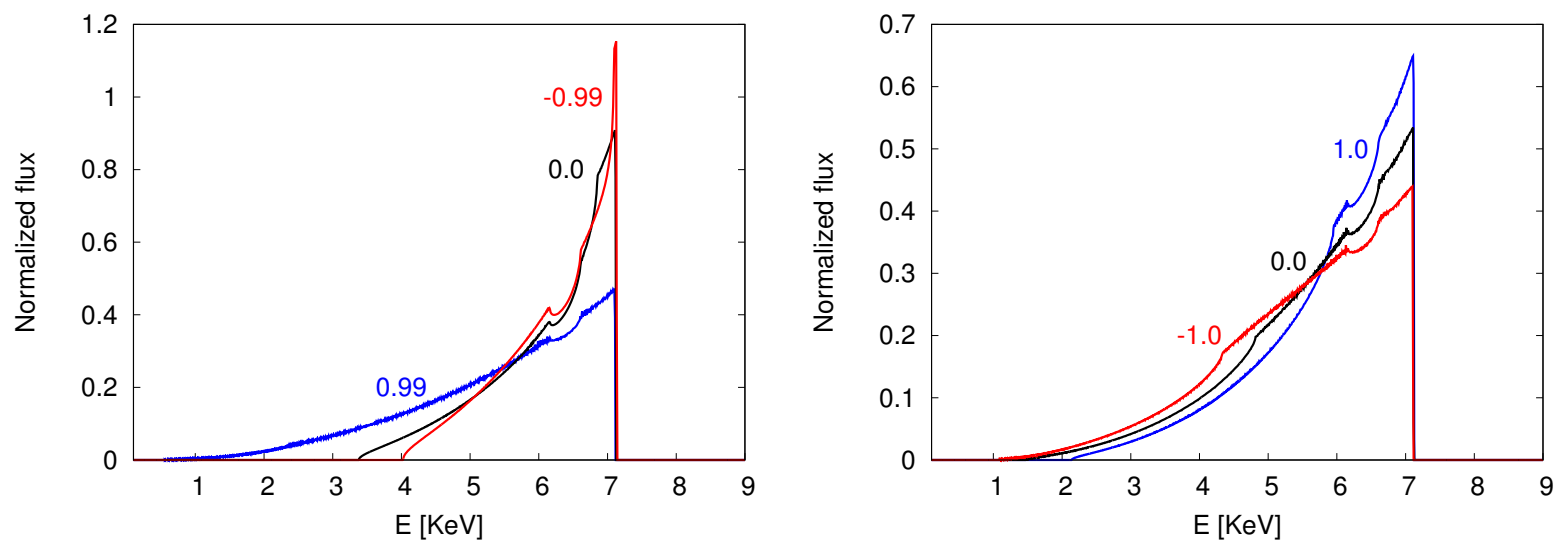

FIG. 2. Normalized flux plotted as a function of energy at the observer using RELLINE_NK. The left figure plots Kerr cases, with the labels indicating the value of $a_{*}$, while the right figure plots non-Kerr cases at a fixed $a_{*}=0.9$, with the labels denoting the value of $\alpha_{13}$. Inclination is fixed in both cases at $45^{\circ}$.

\section{SPECTRAL FITTING AND RESULTS}

We proceed to fit the composite spectrum using our non-Kerr reflection code RELXILL_NK [57, 71]. The Galactic absorption is modeled using TBABS with fixed column density $\left(N_{\mathrm{H}}=4 \times 10^{21} \mathrm{~cm}^{-2}\right)$. For the TBABS model [72], we used the set of interstellar medium abundances of Ref. [73] and the photoelectric cross sections of Ref. [74].

We fit the spectrum to a succession of four models; the final adopted model yields a good fit with $\chi_{\nu}^{2} \sim 1$. Table I provides detailed information on the quality of the fit for each spectrum and each model. In Fig. 3, we show the spectrum residual plots for the progression of the four models, which we now describe.

Model 0: TBABS*POWERlaw. An absorbed powerlaw model, which is clearly deficient, prominently displays the principal reflection features, the Fe K line/edge and Compton hump, in the residuals (Fig. 3).

Model 1: TBABS*RELXILL_NK. A greatly improved fit is achieved by adding our fully relativistic reflection model (RELXILL_NK includes both a power-law and a reflection component). In order to allow for the possibility that the disk might be truncated at $R_{\text {in }}>R_{\mathrm{ISCO}}$, we adopt an extension of the approach of [70] and fix spin to its extreme value of $a_{*}=0.998$, set the deformation parameter to zero, we leave the inner edge of the disk free, and assume the canonical dependence of disk emissivity with radius, namely $\propto r^{-3}$. This model already delivers fits of reasonable quality (Table I). Some pronounced residual features remain. Specifically, two apparent absorption features flank the Fe K line at $\sim 5.6$ and $\sim 7.2$ $\mathrm{keV}$. Other features are also present at higher energies in the region of the Compton hump $(20 \sim 45 \mathrm{keV})$.

Model 2: TBABS*(RELXILL_NK+XILLVER). The residuals are significantly reduced by including an unblurred reflection component via XILLVER. Physically, this reflector could be cold material in a wind or in the

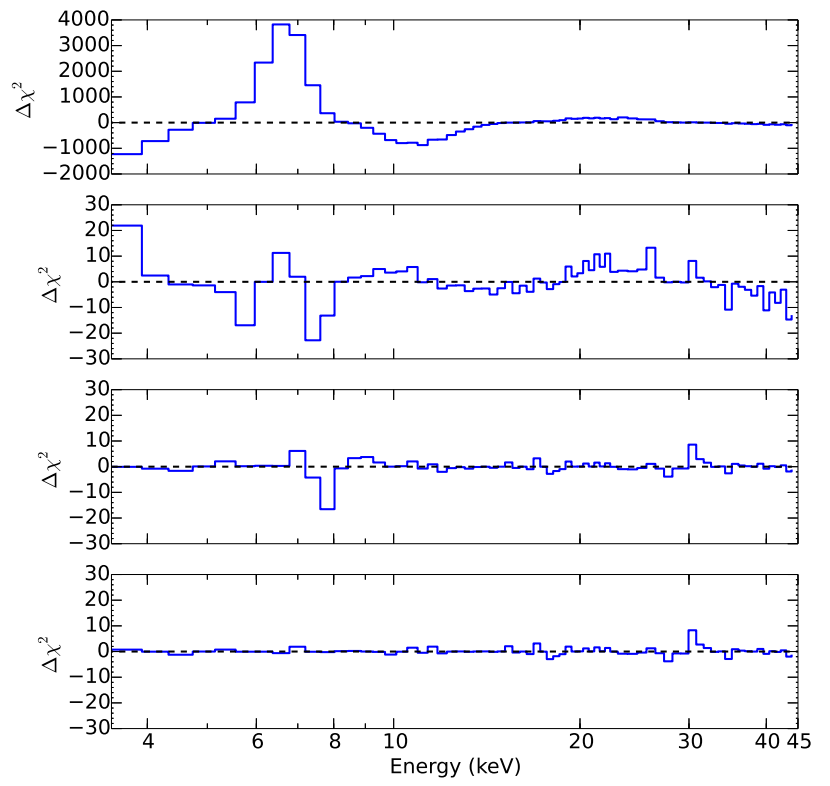

FIG. 3. Contributions to $\chi^{2}$ for the spectrum resulting from fitting a sequence of four models. From top to bottom, the models increase in completeness and performance, starting with an absorbed power-law model to which, incrementally, is added a blurred reflection component (RELXILL_NK); unblurred reflection (XILLVER); and a Gaussian absorption line (GABS).

outer region of a flared disk. The XILLVER parameters which specify the composition and state of gas in the system but far from the black hole are linked to those of RELXILL_NK with one exceptions: the ionization parameter was fixed at its minimum value, $\log \xi=0$. Inclusion of the XILLVER component, which introduces only one new free parameter, namely its normalization, quite 
TABLE I. Statistics of the individual fits to the spectrum.

\begin{tabular}{cccc}
\hline \hline Model & $\chi^{2}$ & $\nu$ & $\chi_{\nu}^{2}$ \\
0 & 24452.29 & 69 & 354.3810 \\
1 & 332.95 & 62 & 5.3701 \\
2 & 101.59 & 61 & 1.6654 \\
3 & 63.91 & 59 & 1.083 \\
\hline
\end{tabular}

significantly improves the fit. While the XILLVER component improves the fit at low energies and in the region of the Compton hump, a strong residual feature remains at $\sim 7.2 \mathrm{keV}$ (Fig. 3) [70].

Model 3: TBABS*(RELXILL_NK+XILLVER)*GABS. Following previous analysis on these data [70], we model the remaining residual feature near $\sim 7.2 \mathrm{keV}$ phenomenologically as absorption using a single Gaussian. As found in previous work, it produces a very good fit to this spectrum, despite its extreme statistical precision $\left(4 \times 10^{7}\right.$ total counts), with an allowance for systematic error of only $0.1 \%$.

Model 3 is the final model we adopt. We make the standard assumption that $R_{\mathrm{in}}=R_{\mathrm{ISCO}}$, as explored previously for this data [70]. This is the key ingredient to constrain the deformation parameter $\alpha_{13}$ and $a_{*}$. The reason is that $R_{\text {in }}$ (set equal to $R_{\mathrm{ISCO}}$ ), $\alpha_{13}$ (which has a significant impact on $R_{\mathrm{ISCO}}$ ) and $a_{*}$ (which maps $R_{\mathrm{ISCO}}$ one to one), are all interconnected and thus have a strong degeneracy waiting to be broken.

Starting from Model 3, we performed Markov Chain Monte-Carlo (MCMC) analysis using the EMCEEHAMMER Python package [75] which implements affineinvariant sampling via the XSPEC implementation described in [76]. With MCMC's great power in highdimensional analysis, we can explore efficiently the parameter space, and directly determine the posterior probabilities for all the model parameters, calculate the confidence contours and search for degeneracies. We utilize 80 walkers, which are initialized in a cluster distributed about the best fit we found in Model 3. Each walker has 870,000 elements in total, and the initial 400,000 elements are discarded as "burn-in" period during which the chain reaches its stationary state. As the autocorrelation length is typically several thousand elements, the net number of independent samples in the parameter space we have is order of $10^{4}$.

Fig. 4 shows the data and the best-fit model (top panel) as well as the different model components (bottom panel). We show a corner plot with all the one and two dimensional projections of the posterior probability distributions of all free parameters in Fig. 5. From the same figure we can also see which parameters are correlated and which are not. In particular, the deformation parameter $\alpha_{13}$ is correlated with the black hole spin $a_{*}$ and the disk's inclination angle $i$, but not much with the other parameters. In Table II, we show the best fit val-

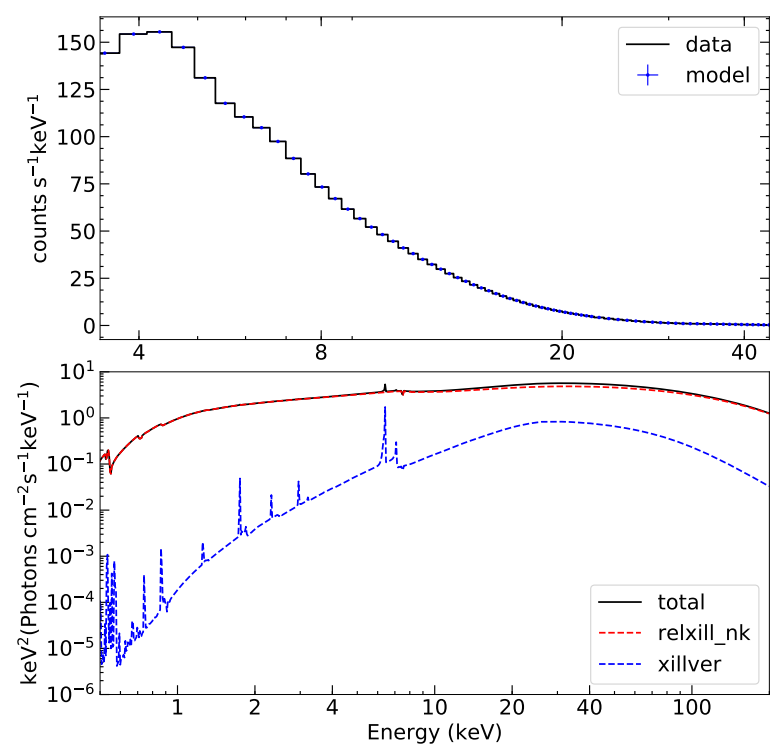

FIG. 4. Data and the best-fit model (top panel) and total spectrum with different model components (bottom panel).

ues with uncertainties for all parameters, based on the marginalized distributions we obtain from the MCMC run. Our results are largely consistent with those found in [70] under the assumption of the Kerr background.

In Fig. 6 we zoom into the $a_{*}$ vs. $\alpha_{13}$ part of Fig. 5 . Several features can be observed here: Firstly, there is some degeneracy between the spin and the deformation parameter, as expected (see Sec. III for a discussion and Fig. 2). In particular, within $90 \%$ confidence $a_{*}=0.92_{-0.12}^{+0.07}$ and $\alpha_{13}=-0.76_{-0.60}^{+0.78}$. Secondly, this result is consistent with previous constraints on $\alpha_{13}$, as reported in $[8,50]$. Thirdly, the Kerr solution $\left(\alpha_{13}=0\right.$, indicated by a solid black line in the figure) is recovered within $90 \%$ confidence. Finally, we notice that the contours of degeneracy obtained here with an MCMC analysis are comparable to those obtained in $[8,50]$. This does not imply that there are no systematic errors, an issue that will be examined further in a separate work.

\section{SUMMARY AND CONCLUSIONS}

In this paper, we have applied the first non-Kerr full Xray relativistic reflection spectral model to test the Kerr nature of an astrophysical black hole. Since we now have more sophisticated reflection models, like the RELXILL family, we have taken a step forward and begun analyzing available X-ray data on specific sources available in archive to demonstrate that tests of the spacetime metric around black holes are possible.

There are currently a number of uncertainties in the astrophysical model limiting the ability to perform accurate and precise tests of the Kerr metric using X-ray reflection spectroscopy. The uncertainty in the corona geometry does not permit to have a robust prediction 
TABLE II. Best fit parameters for Model 3, TBABS*(RELXILL_NK+XILLVER) ${ }^{*}$ GABS, with $R_{\text {in }}=R_{\text {ISCO }}$.

\begin{tabular}{cccc}
\hline \hline Model & Parameter & Value & Unit/Description \\
\hline TBABS & $N_{\mathrm{H}}$ & $4.0 \times 10^{21}$ & Column density $\left(\mathrm{cm}^{-2}\right)$ \\
RELXILL_NK & $R_{\text {in }}$ & 1.0 & Inner radius $\left(R_{\mathrm{ISCO}}\right)$ \\
\hline RELXILL_NK & $a_{*}$ & $0.92_{-0.12}^{+0.07}$ & Spin \\
RELXILL_NK & $i$ & $49 \pm 3$ & Inclination angle (degrees) \\
RELXILL_NK & $\Gamma$ & $1.575_{-0.013}^{+0.014}$ & Power-law index of the incident spectrum \\
RELXILL_NK & $\log \xi$ & $3.37_{-0.09}^{+0.07}$ & Ionization parameter $\left(\mathrm{erg} \cdot \mathrm{cm} \cdot \mathrm{s}^{-1}\right)$ \\
RELXILL_NK & $A_{\mathrm{Fe}}$ & $7.7_{-2.5}^{+2.0}$ & Iron abundance (Solar) \\
RELXILL_NK & $E_{\text {cut }}$ & $85.5_{-3.0}^{+3.2}$ & High energy cutoff $(\mathrm{keV})$ \\
RELXILL_NK & $R_{\mathrm{f}}$ & $0.29_{-0.03}^{+0.04}$ & Reflection fraction \\
RELXILL_NK & $\alpha_{13}$ & $-0.76_{-0.60}^{+0.78}$ & Deformation parameter \\
RELXILL_NK & $N_{\text {relxill_nk }}\left(10^{-3}\right)$ & $35.4_{-0.6}^{+0.9}$ & Normalization of RELXILL_NK \\
XILLVER & $N_{\text {xillver }}\left(10^{-3}\right)$ & $8.3_{-1.6}^{+1.9}$ & Normalization of XILLVER \\
GABS & $E_{\text {line }}$ & $7.40 \pm 0.14$ & Energy of absorption line $(\mathrm{keV})$ \\
GABS & Stength & $0.030_{-0.009}^{+0.013}$ & Line depth \\
\hline & $\chi^{2} /$ d.o.f. & $63.5 / 59=1.08$ & \\
\hline
\end{tabular}

Notes.

Errors are at $90 \%$ confidence level, are statistical only, and are calculated for each parameter from the MCMC after marginalising over all other parameters.

of the emissivity profile for the reflection emission and the commonly adopted power-law or broken power-law emissions are quite crude approximations. The disk is assumed to be infinitesimally thin and the inner edge is set at the ISCO radius, while we know that the disk thickness increases as the mass accretion rate increases and, for high accretion rates, the inner edge of the disk may be at a radius smaller than $R_{\mathrm{ISCO}}$. There are still a number of simplifications in the calculations of the reflection emission in XILLVER: the ionization parameter is constant over radius, the electron density of the disk is fixed and probably too low for some accretion disks, thermal photons from the accretion disk are ignored in the radiative transfer calculations of the reflection emission, etc. Work to remove these simplifications is under way and strictly compulsory if we want to try to use this technique to perform precise tests of GR.

In the present work, we have analyzed a composite spectrum from the detector RXTE PCU-2 on GX 339-4 with the highest observed luminosity. We have only explored the Johannsen deformation parameter $\alpha_{13}$, which has the largest impact on the ISCO radius, and thus on the shape of reflection spectrum, among the deformation parameters of the Johannsen metric. Our final measurement on the black hole spin parameter $a_{*}$ and the Johannsen deformation parameter $\alpha_{13}$ are (at $90 \%$ confidence level)

$$
a_{*}=0.92_{-0.12}^{+0.07}, \quad \alpha_{13}=-0.76_{-0.60}^{+0.78} .
$$

The field is in a nascent state, and despite these being the strongest constraints on Kerr deformation parameters yet, this work is still exploratory and limited by modeling systematics. The avenues for improvement include better data (with new telescopes coming up in next decade), better modeling of the disk, corona and other aspects of the black hole neighborhood (to account for systematic errors), more top-down metrics (for physically relevant deformation parameters) and better bottom-up metrics (for more comprehensive range of deformation parameters), among others.

\section{ACKNOWLEDGMENTS}

J.W. thanks the Cahill Center for Astronomy and Astrophysics at Caltech for support and hospitality during her visit. This work was supported by the National Natural Science Foundation of China (NSFC), Grant No. U1531117, and Fudan University, Grant No. IDH1512060. A.B.A. also acknowledges the support from the Shanghai Government Scholarship (SGS). C.B. and J.A.G. also acknowledge support from the Alexander von Humboldt Foundation. S.N. acknowledges support from the Excellence Initiative at Eberhard-Karls Universität Tübingen. J.F.S. has been supported by NASA Einstein Fellowship Grant PF5-160144. 


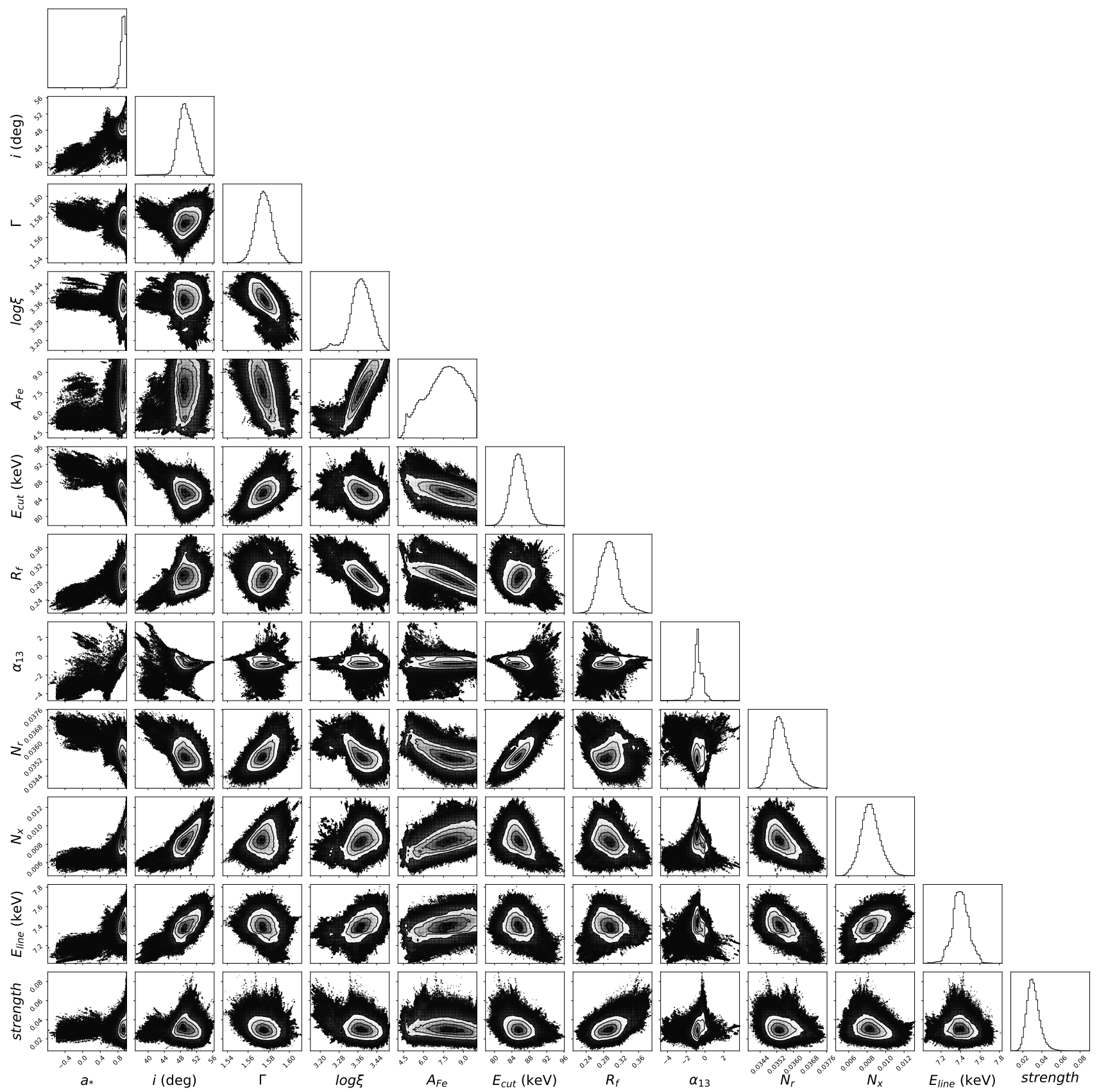

FIG. 5. 1-, 2-, 3- $\sigma$ confidence contours for all the free parameters in Model 3 after the MCMC run.

[1] A. Einstein, The Foundation of the General Theory of Relativity, Annalen Phys. 49, no. 7, 769 (1916) [Annalen Phys. 14, 517 (2005)].

[2] C. M. Will, The Confrontation between General Relativity and Experiment, Living Rev. Rel. 17, 4 (2014) [arXiv:1403.7377 [gr-qc]].

[3] S. DeDeo and D. Psaltis, Towards New Tests of Strongfield Gravity with Measurements of Surface Atomic Line Redshifts from Neutron Stars, Phys. Rev. Lett. 90,
141101 (2003) [astro-ph/0302095].

[4] T. Johannsen, D. Psaltis and J. E. McClintock, Constraints on the Size of Extra Dimensions from the Orbital Evolution of Black-Hole X-Ray Binaries, Astrophys. J. 691, 997 (2009) [arXiv:0803.1835 [astro-ph]].

[5] L. Kong, Z. Li and C. Bambi, Constraints on the spacetime geometry around 10 stellar-mass black hole candidates from the disk's thermal spectrum, Astrophys. J. 797, 78 (2014) [arXiv:1405.1508 [gr-qc]]. 


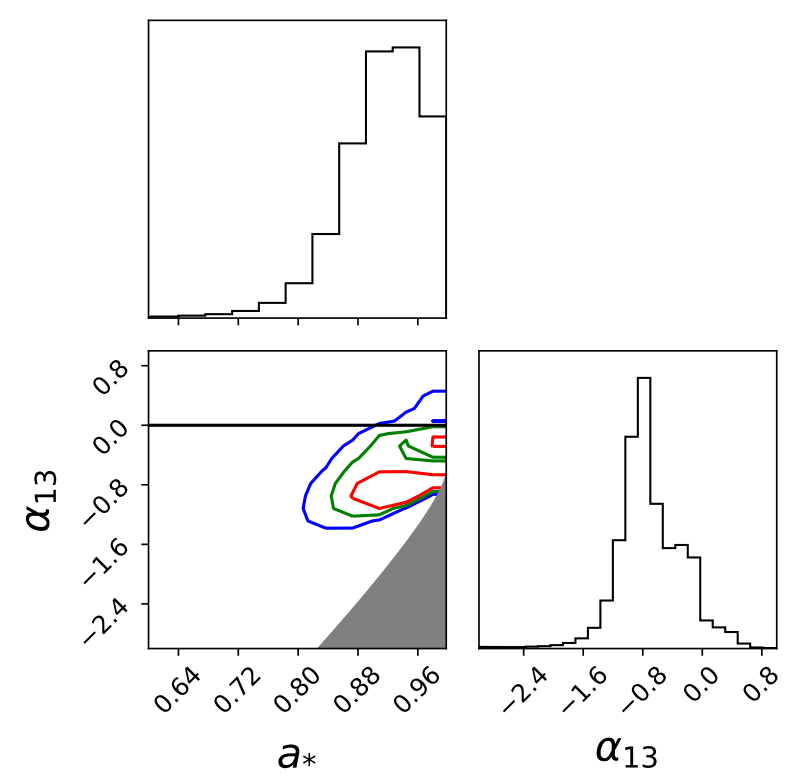

FIG. 6. 1-, 2-, 3- $\sigma$ confidence contours for $a_{*}$ and $\alpha_{13}$ in Model 3 after the MCMC run.

[6] N. Yunes, K. Yagi and F. Pretorius, Theoretical Physics Implications of the Binary Black-Hole Mergers GW150914 and GW151226, Phys. Rev. D 94, 084002 (2016) [arXiv:1603.08955 [gr-qc]].

[7] T. Johannsen, C. Wang, A. E. Broderick, S. S. Doeleman, V. L. Fish, A. Loeb and D. Psaltis, Testing General Relativity with Accretion-Flow Imaging of Sgr $A$ *, Phys. Rev. Lett. 117, 091101 (2016) [arXiv:1608.03593 [astro-ph.HE]].

[8] Z. Cao, S. Nampalliwar, C. Bambi, T. Dauser and J. A. Garcia, Testing general relativity with the reflection spectrum of the supermassive black hole in 1H0707-495, Phys. Rev. Lett. 120, 051101 (2018) [arXiv:1709.00219 [gr-qc]].

[9] P. S. Joshi and D. Malafarina, Recent developments in gravitational collapse and spacetime singularities, Int. J. Mod. Phys. D 20, 2641 (2011) [arXiv:1201.3660 [gr-qc]].

[10] E. Maoz, Dynamical constraints on alternatives to massive black holes in galactic nuclei, Astrophys. J. 494, L181 (1998) [astro-ph/9710309].

[11] B. P. Abbott et al. [LIGO Scientific and Virgo Collaborations], Observation of Gravitational Waves from a Binary Black Hole Merger, Phys. Rev. Lett. 116, 061102 (2016) [arXiv:1602.03837 [gr-qc]].

[12] B. P. Abbott et al. [LIGO Scientific and Virgo Collaborations], GW151226: Observation of Gravitational Waves from a 22-Solar-Mass Binary Black Hole Coalescence, Phys. Rev. Lett. 116, 241103 (2016) [arXiv:1606.04855 [gr-qc]].

[13] R. P. Kerr, Gravitational field of a spinning mass as an example of algebraically special metrics, Phys. Rev. Lett. 11, 237 (1963).

[14] E. T. Newman, R. Couch, K. Chinnapared, A. Exton, A. Prakash and R. Torrence, Metric of a Rotating, Charged Mass, J. Math. Phys. 6, 918 (1965).

[15] W. Israel, Event horizons in static vacuum space-times,
Phys. Rev. 164, 1776 (1967).

[16] B. Carter, Axisymmetric Black Hole Has Only Two Degrees of Freedom, Phys. Rev. Lett. 26, 331 (1971).

[17] D. C. Robinson, Uniqueness of the Kerr black hole, Phys. Rev. Lett. 34, 905 (1975).

[18] P. T. Chrusciel, J. L. Costa and M. Heusler, Stationary Black Holes: Uniqueness and Beyond, Living Rev. Rel. 15, 7 (2012) [arXiv:1205.6112 [gr-qc]].

[19] R. H. Price, Nonspherical perturbations of relativistic gravitational collapse. 1. Scalar and gravitational perturbations, Phys. Rev. D 5, 2419 (1972).

[20] R. H. Price, Nonspherical Perturbations of Relativistic Gravitational Collapse. II. Integer-Spin, Zero-Rest-Mass Fields, Phys. Rev. D 5, 2439 (1972).

[21] C. Bambi, A. D. Dolgov and A. A. Petrov, Black holes as antimatter factories, JCAP 0909, 013 (2009) [arXiv:0806.3440 [astro-ph]].

[22] E. Barausse, V. Cardoso and P. Pani, Can environmental effects spoil precision gravitational-wave astrophysics?, Phys. Rev. D 89, 104059 (2014) [arXiv:1404.7149 [grqc]].

[23] C. Bambi, D. Malafarina and N. Tsukamoto, Note on the effect of a massive accretion disk in the measurements of black hole spins, Phys. Rev. D 89, 127302 (2014) [arXiv:1406.2181 [gr-qc]].

[24] E. Berti et al., Testing General Relativity with Present and Future Astrophysical Observations, Class. Quant. Grav. 32, 243001 (2015) [arXiv:1501.07274 [gr-qc]].

[25] G. Dvali and C. Gomez, Black Hole's Quantum NPortrait, Fortsch. Phys. 61, 742 (2013) [arXiv:1112.3359 [hep-th]].

[26] G. Dvali and C. Gomez, Black Hole's 1/N Hair, Phys. Lett. B 719, 419 (2013) [arXiv:1203.6575 [hep-th]].

[27] S. B. Giddings, Possible observational windows for quantum effects from black holes, Phys. Rev. D 90, 124033 (2014) [arXiv:1406.7001 [hep-th]].

[28] C. A. R. Herdeiro and E. Radu, Kerr black holes with scalar hair, Phys. Rev. Lett. 112, 221101 (2014) [arXiv:1403.2757 [gr-qc]].

[29] C. A. R. Herdeiro and E. Radu, Asymptotically flat black holes with scalar hair: a review, Int. J. Mod. Phys. D 24, 1542014 (2015) [arXiv:1504.08209 [gr-qc]].

[30] C. Bambi, Testing black hole candidates with electromagnetic radiation, Rev. Mod. Phys. 89, 025001 (2017) [arXiv:1509.03884 [gr-qc]].

[31] C. Bambi, J. Jiang and J. F. Steiner, Testing the nohair theorem with the continuum-fitting and the iron line methods: a short review, Class. Quant. Grav. 33, 064001 (2016) [arXiv:1511.07587 [gr-qc]].

[32] T. Johannsen, Testing the No-Hair Theorem with Observations of Black Holes in the Electromagnetic Spectrum, Class. Quant. Grav. 33, 124001 (2016) [arXiv:1602.07694 [astro-ph.HE]].

[33] N. Yunes and X. Siemens, Gravitational-Wave Tests of General Relativity with Ground-Based Detectors and Pulsar Timing-Arrays, Living Rev. Rel. 16, 9 (2013) [arXiv:1304.3473 [gr-qc]].

[34] K. Yagi and L. C. Stein, Black Hole Based Tests of General Relativity, Class. Quant. Grav. 33, no. 5, 054001 (2016) [arXiv:1602.02413 [gr-qc]].

[35] S. N. Zhang, W. Cui and W. Chen, Black hole spin in X-ray binaries: Observational consequences, Astrophys. J. 482, L155 (1997) [astro-ph/9704072].

[36] J. E. McClintock et al., Measuring the Spins of Accret- 
ing Black Holes, Class. Quant. Grav. 28, 114009 (2011) [arXiv:1101.0811 [astro-ph.HE]].

[37] J. E. McClintock, R. Narayan and J. F. Steiner, Black Hole Spin via Continuum Fitting and the Role of Spin in Powering Transient Jets, Space Sci. Rev. 183, 295 (2014) [arXiv:1303.1583 [astro-ph.HE]].

[38] L. W. Brenneman and C. S. Reynolds, Constraining Black Hole Spin Via X-ray Spectroscopy, Astrophys. J. 652, 1028 (2006) [astro-ph/0608502].

[39] C. S. Reynolds, Measuring Black Hole Spin using X-ray Reflection Spectroscopy, Space Sci. Rev. 183, 277 (2014) [arXiv:1302.3260 [astro-ph.HE]].

[40] L. Brenneman, Measuring Supermassive Black Hole Spins in Active Galactic Nuclei, (Springer, New York, 2013) [arXiv:1309.6334 [astro-ph.HE]].

[41] J. Schee and Z. Stuchlik, Optical phenomena in the field of braneworld Kerr black holes, Int. J. Mod. Phys. D 18, 983 (2009) [arXiv:0810.4445 [astro-ph]].

[42] D. F. Torres, Accretion disc onto a static nonbaryonic compact object, Nucl. Phys. B 626, 377 (2002) [hep$\mathrm{ph} / 0201154]$.

[43] C. Bambi and E. Barausse, Constraining the quadrupole moment of stellar-mass black-hole candidates with the continuum fitting method, Astrophys. J. 731, 121 (2011) [arXiv:1012.2007 [gr-qc]].

[44] T. Johannsen and D. Psaltis, Testing the No-Hair Theorem with Observations in the Electromagnetic Spectrum. IV. Relativistically Broadened Iron Lines, Astrophys. J. 773, 57 (2013) [arXiv:1202.6069 [astro-ph.HE]].

[45] C. Bambi, A code to compute the emission of thin accretion disks in non-Kerr space-times and test the nature of black hole candidates, Astrophys. J. 761, 174 (2012) [arXiv:1210.5679 [gr-qc]].

[46] C. Bambi, Testing the space-time geometry around black

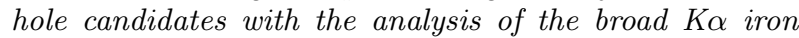
line, Phys. Rev. D 87, 023007 (2013) [arXiv:1211.2513 [gr-qc]].

[47] M. Zhou, A. Cardenas-Avendano, C. Bambi, B. Kleihaus and J. Kunz, Search for astrophysical rotating Ellis wormholes with $X$-ray reflection spectroscopy, Phys. Rev. D 94, 024036 (2016) [arXiv:1603.07448 [gr-qc]].

[48] Y. Ni, M. Zhou, A. Cardenas-Avendano, C. Bambi,

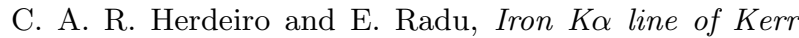
black holes with scalar hair, JCAP 1607, 049 (2016) [arXiv:1606.04654 [gr-qc]].

[49] J. Jiang, C. Bambi and J. F. Steiner, Testing the Kerr Nature of Black Hole Candidates using Iron Line Spectra in the CPR Framework, Astrophys. J. 811, 130 (2015) [arXiv:1504.01970 [gr-qc]].

[50] A. Tripathi, S. Nampalliwar, A. B. Abdikamalov, D. Ayzenberg, J. Jiang and C. Bambi, Testing the Kerr nature of the supermassive black hole in Ark 564, arXiv:1804.10380 [gr-qc].

[51] B. Bertotti, L. Iess and P. Tortora, A test of general relativity using radio links with the Cassini spacecraft, Nature 425, 374 (2003).

[52] J. G. Williams, S. G. Turyshev and D. H. Boggs, Progress in lunar laser ranging tests of relativistic gravity, Phys. Rev. Lett. 93, 261101 (2004) [gr-qc/0411113].

[53] T. Johannsen and D. Psaltis, A Metric for Rapidly Spinning Black Holes Suitable for Strong-Field Tests of the No-Hair Theorem, Phys. Rev. D 83, 124015 (2011) [arXiv:1105.3191 [gr-qc]].

[54] T. Johannsen, Regular Black Hole Metric with Three
Constants of Motion, Phys. Rev. D 88, 044002 (2013) [arXiv:1501.02809 [gr-qc]].

[55] R. Konoplya, L. Rezzolla and A. Zhidenko, General parametrization of axisymmetric black holes in metric theories of gravity, Phys. Rev. D 93, 064015 (2016) [arXiv:1602.02378 [gr-qc]].

[56] C. Bambi, Astrophysical Black Holes: A Compact Pedagogical Review, Annalen Phys. 530, 1700430 (2018) [arXiv:1711.10256 [gr-qc]].

[57] C. Bambi, A. Cardenas-Avendano, T. Dauser, J. A. Garcia and S. Nampalliwar, Testing the Kerr black hole hypothesis using X-ray reflection spectroscopy, Astrophys. J. 842, 76 (2017) [arXiv:1607.00596 [gr-qc]].

[58] K. A. Arnaud, Astronomical Data Analysis Software and Systems V, 101, 17 (1996).

[59] R. R. Ross and A. C. Fabian, A Comprehensive range of $x$-ray ionized reflection models, Mon. Not. Roy. Astron. Soc. 358, 211 (2005) [astro-ph/0501116].

[60] J. Garcia, T. R. Kallman and R. F. Mushotzky, X-ray reflected spectra from accretion disk models.II. Diagnostic tools for X-ray observations, Astrophys. J. 731, 131 (2011) [arXiv:1101.1115 [astro-ph.HE]].

[61] J. Garcia, T. Dauser, C. S. Reynolds, T. R. Kallman, J. E. McClintock, J. Wilms and W. Eikmann, X-ray reflected spectra from accretion disk models. III. A complete grid of ionized reflection calculations, Astrophys. J. 768, 146 (2013) [arXiv:1303.2112 [astro-ph.HE]].

[62] J. García et al., Improved Reflection Models of BlackHole Accretion Disks: Treating the Angular Distribution of X-rays, Astrophys. J. 782, 76 (2014) [arXiv:1312.3231 [astro-ph.HE]].

[63] T. Dauser, J. Wilms, C. S. Reynolds and L. W. Brenneman, Broad emission lines for negatively spinning black holes, Mon. Not. Roy. Astron. Soc. 409, 1534 (2010) [arXiv:1007.4937 [astro-ph.HE]].

[64] T. Dauser, J. Garcia, J. Wilms, M. Bock, L. W. Brenneman, M. Falanga, K. Fukumura and C. S. Reynolds, Irradiation of an Accretion Disc by a Jet: General Properties and Implications for Spin Measurements of Black Holes, Mon. Not. Roy. Astron. Soc. 430, 1694 (2013) [arXiv:1301.4922 [astro-ph.HE]].

[65] T. Dauser, J. Garcia, M. L. Parker, A. C. Fabian and J. Wilms, The role of the reflection fraction in constraining black hole spin, Mon. Not. Roy. Astron. Soc. 444, 100 (2014) [arXiv:1408.2347 [astro-ph.HE]].

[66] T. Kallman and M. Bautista, Photoionization and HighDensity Gas, Astrophys. J. Suppl. 133, 221 (2001).

[67] C. Bambi, L. Modesto and L. Rachwa?, Spacetime completeness of non-singular black holes in conformal gravity, JCAP 1705, 003 (2017) [arXiv:1611.00865 [gr-qc]].

[68] M. Zhou, Z. Cao, A. Abdikamalov, D. Ayzenberg, C. Bambi, L. Modesto and S. Nampalliwar, Testing conformal gravity with the supermassive black hole in 1H0707-495, arXiv:1803.07849 [gr-qc].

[69] J. A. García, J. E. McClintock, J. F. Steiner, R. A. Remillard and V. Grinberg, An Empirical Method for Improving the Quality of RXTE PCA Spectra, Astrophys. J. 794, 73 (2014) [arXiv:1408.3607 [astro-ph.HE]].

[70] J. A. García, J. F. Steiner, J. E. McClintock, R. A. Remillard, V. Grinberg and T. Dauser, X-ray Reflection Spectroscopy of the Black Hole GX 3394: Exploring the Hard State With Unprecedented Sensitivity, Astrophys. J. 813, 84 (2015) [arXiv:1505.03607 [astro-ph.HE]].

[71] J. García et al., Improved Reflection Models of Black- 
Hole Accretion Disks: Treating the Angular Distribution of X-rays, Astrophys. J. 782, 76 (2014) [arXiv:1312.3231 [astro-ph.HE]].

[72] J. Wilms, A. Allen and R. McCray, On the Absorption of X-rays in the interstellar medium, Astrophys. J. 542, 914 (2000) [astro-ph/0008425].

[73] E. Anders and N. Grevesse, Abundances of the elements: Meteroritic and solar, Geochim. Cosmochim. Acta 53, 197 (1989).

[74] D. A. Verner, G. J. Ferland, K. T. Korista and
D. G. Yakovlev, Atomic data for astrophysics. 2. New analytic FITS for photoionization cross-sections of atoms and ions, Astrophys. J. 465, 487 (1996) [astro$\mathrm{ph} / 9601009]$.

[75] D. Foreman-Mackey, D. W. Hogg, D. Lang and J. Goodman, emcee: The MCMC Hammer, Publ. Astron. Soc. Pac. 125, 306 (2013) [arXiv:1202.3665 [astro-ph.IM]].

[76] J. F. Steiner et al., A Broad Iron Line in LMC X-1, Mon. Not. Roy. Astron. Soc. 427, 2552 (2012) [arXiv:1209.3269 [astro-ph.HE]]. 